\title{
O TRABALHO DA MULHER PESCADORA: UM ESTUDO DE CASO NO ESTADO DO PARÁ*
}

\section{THE WORK OF FISHERWOMEN: A CASE STUDY IN THE STATE OF PARÁ}

\author{
Jessica dos Santos Lima Pantoja ${ }^{1 *}$, Amanda Victória Botelho da Silva ${ }^{2}$, Marcela Cardoso Sena ${ }^{3}$, \\ Sâmela Carneiro Gomes ${ }^{4} \&$ Rafael Anaisce das Chagas ${ }^{5}$
}

\author{
${ }^{1,2,3,4}$ Curso de Engenharia de Pesca da Universidade Federal Rural da Amazônia - UFRA \\ ${ }^{5}$ Instituto Socioambiental e dos Recursos Hídricos, Universidade Federal Rural da Amazônia - UFRA \\ *E-mail: *jessica.ufra28@gmail.com
}

Recebido em 7 de agosto de 2021 - Publicado em 2 de novembro de 2021

\begin{abstract}
Resumo O presente trabalho teve como objetivo revisar a literatura sobre estudos realizados com as mulheres pescadoras no estado do Pará, a saber suas condições socioeconômica e o papel que desempenham na pesca artesanal. A busca de dados foi realizada em duas plataformas o Google Acadêmico e o BDTD, utilizando critérios de exclusão e inclusão e com uso de operadores lógicos. Os critérios de inclusão utilizados foram: i) trabalhos somente com mulheres pescadoras que foram desenvolvidos no estado do Pará; ii) trabalhos que foram publicados nos últimos vinte anos; iii) trabalhos originais, podendo ser artigos, dissertações e/ou teses; iv) trabalhos que mostrem o perfil socioeconômico das mulheres pescadoras no Pará; v) trabalhos que mostrem o papel da mulher na pesca artesanal. Os critérios de exclusão foram: i) trabalhos publicados antes do ano de 2001; ii) trabalhos de revisão bibliográfica e iii) trabalhos sobre mulheres pescadoras, mas que não foram desenvolvidos no estado do Pará. A pesquisa mostrou que os trabalhos publicados são recentes, grande parte publicado nos últimos 15 anos e a maioria estão em versão de artigos científicos, disponível no Google Acadêmico. As pesquisas foram realizadas principalmente no nordeste paraense e poucos são os trabalhos que mostram o perfil econômico e social das mulheres pescadoras. Apesar de exercerem um papel importante na pesca artesanal, principalmente no processamento do pescado, o trabalho das mulheres pescadoras ainda não é reconhecido e essas ainda estão invisíveis aos olhos da sociedade.
\end{abstract}

Palavras-chave: pesca na Amazônia; pescadora; trabalho feminino

\begin{abstract}
This study aimed to review the literature on studies carried out with women fishers in the state of Pará, namely their socioeconomic conditions and the role they play in artisanal fishing. The data search was performed on two platforms Google Scholar and BDTD, using exclusion and inclusion criteria and using logical operators. The inclusion criteria used were: i) works only with female fishers that were developed in the state of Pará; ii) works that were published in the last twenty years; iii) original works, which may be articles, dissertations and/or theses; iv) works that show the socioeconomic profile of female fishers in Pará; v) works that show the role of women in artisanal fishing. Exclusion criteria were: i) works published before 2001; ii) bibliographic review works and iii) works on fisherwomen, but which were not developed in the state of Pará, available on Google Scholar. The surveys were carried out mainly in the northeast of Pará and there are few works that show the economic and social profile of female fishermen. Despite playing an important role in artisanal fishing, especially in fish processing, the work of women fishers is still not recognized and they are still invisible to society.
\end{abstract}

Key words: Amazon fishing; fisherwomen; woman work 


\section{Introdução}

As atividades de pesca são de extrema importância, pois desempenha um importante papel social e econômico na vida de milhões de pessoas no Brasil e no mundo. Da mesma forma na Amazônia existe uma grande dependência da população ribeirinha nessa atividade, visto que, muitos são pescadores, e tem a pesca como sua fonte de renda e fonte de alimentação (Silva et al., 2014)

A pesca artesanal na região amazônica é realizada em regime familiar, onde o trabalho é dividido entre marido, mulher e filhos, ou seja, todos os membros da família estão envolvidos no seu desenvolvimento (Almeida, 2013). Nesse cenário a mulher desempenha um papel preponderante no processo produtivo. No entanto, o trabalho que as mulheres pescadoras exercem na pesca ainda não é reconhecido e estão "invisíveis" aos olhos da sociedade dita moderna (Sousa, 2018). Em grande parte, isso ocorre, porque a pesca ainda é vista como uma profissão de domínio masculino e muitos interpretam o papel dessas mulheres pescadoras na cadeia produtiva como um trabalho secundário ou de apenas ajudantes de seus maridos (Araújo \& Milani, 2016)

A pesca artesanal é uma atividade exercida primordialmente por homens, sempre considerado responsável pelo sustento familiar, cuja normas sociais ordenam o espaço doméstico à atuação feminina, gerando a desigualdade de gênero (Sorj, 2010). Sousa et al. (2018) ressaltam que somente no início dos anos 2000 as mulheres pescadoras puderam ser contempladas por instrumentos de políticas públicas, tais como, o seguro defeso.

De acordo com a Federação dos Sindicatos de Pescadores do Estado do Pará (Fespapa), o número de pescadores artesanais no estado é de aproximadamente 12 mil. Na região do Baixo Amazonas (Pará), atualmente existem 13 colônias de pescadores das quais sete entidades possuem mulheres entre os dirigentes, e em três colônias exercem o cargo de presidente. Em Santarém, desde 1982 as mulheres conquistaram espaço na direção da colônia de Pescadores Z-20, tendo em 2017 uma pescadora a assumir a direção de finanças e patrimônio da entidade (Pinto, 2016).

Neste contexto, o presente trabalho teve como objetivo revisar a literatura científica sobre os estudos realizados com o tema "mulheres pescadoras no estado do Pará", e com isso inferir acerca de suas condições socioeconômicas e o papel que desempenham na atividade da pesca artesanal.

\section{Material e Métodos}

A primeira fase da revisão sistemática foi a busca das literaturas cientificas nas bases de dados selecionadas. Foram selecionadas duas bases de dados, o Google Acadêmico (https://scholar.google.com.br/?hl=pt) e o website da Biblioteca Digital Brasileira de Teses e Dissertações BDTD (https://bdtd.ibict.br/vufind/). O uso das bases de dados selecionadas justifica-se pois o Google Acadêmico é uma base de dados consistente em uma ampla gama de artigos científicos sobre o assunto abordado. Enquanto a BDTD possui uma boa quantidade de trabalhos sobre o tema, na forma de teses e dissertações.

Os critérios de seleção de literatura científica foram definidos a seguir: (Pesca* OR mulher*) and (Pará OR paraense OR “estado do Pará"). O uso de * possibilita a inclusão de palavras derivadas. Os critérios de inclusão adotados foram: i) trabalhos somente com mulheres pescadoras que foram desenvolvidos no estado do Pará; ii) trabalhos que foram publicados nos últimos vinte anos; trabalhos originais, podendo ser artigos, dissertações e/ou teses; iv) trabalhos que mostrem o perfil socioeconômico das mulheres pescadoras no Pará; v) trabalhos que mostrem o papel da mulher na pesca artesanal. Os critérios de exclusão adotados foram: i) trabalhos publicados antes do ano 2001; ii) trabalhos de revisão bibliográfica; iii) trabalhos sobre mulheres pescadoras, mas que não foram desenvolvidos no estado do Pará.

Através desse procedimento de busca foram identificadas inicialmente 7.984 publicações com potencial elegível. Após a primeira análise, com avaliação dos títulos, no total 37 publicações (Google Acadêmico = 24 artigos; três dissertações e uma tese) e (BDTD = seis dissertações e uma tese) foram considerados elegíveis para a segunda fase da revisão, que constituiu a leitura dos resumos. Após a avaliação dos resumos, os trabalhos que que pareciam preencher os critérios de inclusão foram lidos na íntegra. Nessa etapa, 17 trabalhos atenderam todos os critérios de inclusão, sendo que duas dissertações estavam no Google Acadêmico e no BDTD.

Na avaliação dos artigos, foram observados os seguintes aspectos:

- Amostra (dimensionamento adequado e envolvendo a participação de mulheres pescadoras)

- Instrumento (metodologia ou ferramenta utilizada para a obtenção dos dados)

- Periódico no qual os artigos selecionados foram publicados (classificados conforme o critério Qualis proposto pela Capes (2013-16). 


\section{Resultados e Discussão}

As análises da literatura encontrada sobre os estudos realizados com o tema "mulheres pescadoras no estado do Pará" indicam que os trabalhos foram publicados entre os períodos de 2006 a 2021. Esse período destaca que mesmo considerando 20 anos de publicações cientificas, apenas nos 15 anos anteriores foram evidenciadas pesquisas com o tema proposto.

Na tabela 1 é apresentado as informações dos trabalhos selecionados e, é possível observar que a maioria foram realizados no Nordeste Paraense, principalmente nos municípios de Bragança e na ilha do Marajó. Pesquisas também foram realizadas em três RESEX marinhas: (RESEX-Mar de São João da Ponta; RESEX-Mar de Caeté/Bragança e RESEX-Mar Mãe Curuçá). Todos os trabalhos utilizaram o instrumento, aplicação de questionário, para a obtenção dos dados e o dimensionamento da amostra foi adequado e com participação somente de mulheres.

Dez trabalhos foram publicados em revistas que não possuem registro em banco de dados da Capes (Azevedo, 2016; Araújo, 2016; Oliveira, 2019; Sousa, 2018; Araujo, 2015; Costa, 2015; Rodrigues, 2018; Machado, 2007; Rocha, 2011; Oliveira, 2018). Das revistas registradas para a verificação da Qualis duas foram publicadas em periódicos com Qualis A3 (Costa, 2020; Guedes, 2021), três com Qualis B3 (Sousa, 2018; Rodrigues, 2018; Simonian, 2006) e duas com Qualis C (Silva, 2014; Rodrigues, 2017).

Com base em todos os trabalhos selecionados e no critério de inclusão, o perfil socioeconômico das mulheres pescadoras do estado do Pará é que essas possuem em média 38 anos, quatros filhos/pescadora, $80 \%$ das mulheres entrevistadas eram casadas e possuem baixo nível de escolaridade, ensino fundamental incompleto. A maioria (aproximadamente 90\%) das mulheres pescadoras entrevistadas nos trabalhos, tinham como a atividade pesqueira a única atividade para obtenção de renda, sendo bem poucas que exerciam outras atividades além da pesca, como a venda de cosméticos, por exemplo, devido aos seus afazeres domésticos e em média trabalhavam 10 horas por dia.

Os trabalhos de Rodrigues (2018), realizado na comunidade da Salvação no município de Alenquer, de Simonian (2006) na ilha de Trambioca (Barcarena) e de Silva et al. (2014) na jusante da Usina Hidrelétrica de Tucuruí, todos no estado do Pará, mostram a mulheres pescadoras envolvidas na pesca do camarão amazônico Macrobrachium amazonicum (Helle, 1862). Em tais locais, as mulheres estão presentes em todo o processo da cadeia produtiva, desde a captura, beneficiamento e comercialização. Enquanto o trabalho de Araujo (2015) mostra a mulher na cadeia produtiva do mexilhão Mytella charruana (d' Orbigny, 1846) e os demais trabalhos, as mulheres pescadoras estão presentes na pesca do caranguejo-uçá Ucides cordatus (Linnaeus, 1763), principalmente na extração da massa.

O trabalho de Araujo (2018), além de mostrar o perfil socioeconômico das mulheres pescadoras em Caratateua/Bragança, mostrou que as pescadoras artesanais dessa região estão expostas ao Mercúrio e glutationa, pois as mulheres que participaram do experimento obtiveram resultados de concentrações desses metais em níveis elevados.

Foi observado que ainda são poucos os estudos que abordam o perfil socioeconômico das mulheres pescadoras no Pará e o papel que exercem na atividade pesqueira. No entanto, segundo os trabalhos encontrados, é possível afirmar que após 2010 as mulheres assumiram mais responsabilidades no desenvolvimento da pesca artesanal. Essa afirmação advém das mobilizações em com objetivo de garantir equidade no acesso às políticas públicas, na seguridade social e previdenciária direcionadas no setor pesqueiro. Isso de uma certa forma ajudou a diminuir as desigualdades históricas existentes, dando uma visibilidade maior na inserção do sexo feminino na atividade pesqueira (Sousa et al., 2018). Porém, todos os trabalhos selecionados mostraram que por mais as pescadoras artesanais tenham ganhado espaço, a falta de reconhecimento ainda é grande e seu trabalho ainda é invisível para a sociedade.

Além disso, mesmo as pescadoras terem conseguido alguns avanços dentro da atividade pesqueira, a invisibilidade de sua produção ainda é um entrave que não foi vencido, principalmente dentro de suas entidades representativas de classes, a exemplo das diretorias das Colônias de Pescadores que, em sua maioria, são ocupadas por homens (Sousa et al., 2018).

A maioria dos trabalhos realizados sobre as mulheres pescadoras no estado do Pará concerne sobre a desigualdade de gênero, e esses são discussões e debates (Souza et al, 2017; Lima \& Mota, 2016). Em minoria elas mostram a sua realidade e a sua importância na pesca artesanal, o que pode ser observado pelo número de trabalhos que foram selecionados para a revisão do trabalho. 


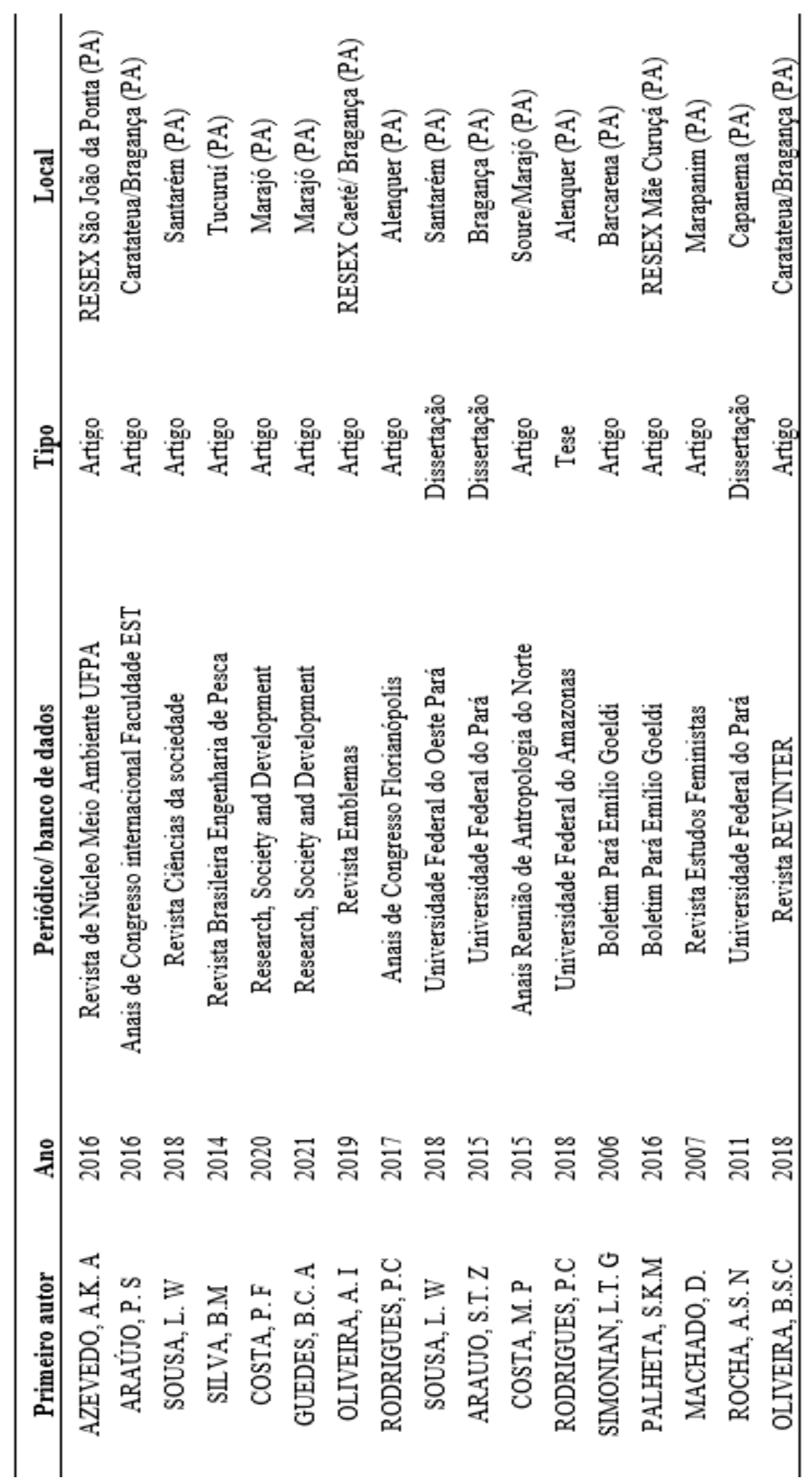

Os trabalhos demostraram que as mulheres pescadoras exercem um importante papel na pesca artesanal, em sua maioria são responsáveis pelo beneficiamento do pescado e sua comercialização (Simonian, 2006; Araujo, 2015). Algumas pescadoras realizam a captura de mariscos e pescarias em regiões próximas a comunidade e com uso de canoas. A maiorias dessas pescadoras estão envolvidas na cadeia de produção do caranguejo-uçá (Ucides cordatus) e realizam a extração da massa do caranguejo (Oliveira, 2019; Araújo \& Milani, 2016). Os estudos mostraram que o 
trabalho dessas mulheres é visto como secundário, pelo fato de não realizarem a pesca em si, mas estarem mais presente em outros processos da cadeia de produção.

Dessa forma, elas são vistas mais como ajudantes, tanto que o termo "pescadora artesanal" ainda é aceito com ressalva, enquanto os termos "marisqueiras", "catadoras" e até "extrativista" são mais utilizados e parecem ser mais aceito pela sociedade (Oliveira, 2019). Esses estudos também mostraram que as condições das mulheres pescadoras de vida e de trabalho, é precária (Azevedo \& Moraes, 2016; Araújo \& Milani, 2016). Muitas pescadoras relatam que exercem essa profissão por não ter oportunidade de conseguir outra profissão, devido ao baixo nível de escolaridade e porque necessita complementar a renda familiar que é muito baixa (Machado, 2007).

Em alguns estados do Brasil, a situação das mulheres pescadoras não difere muito dos resultados encontrado no estado do Pará. Sá-oliveira et al. (2013) em seu trabalho realizado na Usina Hidrelétrica de Coaracy Nunes, no estado do Amapá relata que as mulheres pescadoras são responsáveis pela atividade de limpeza e comercialização do pescado e existe um baixo percentual de participação delas na pesca (em torno de 15\%), e elas tem um papel pouco valorizado nas pesquisas de estatísticas pesqueiras. O trabalho de Ferreira et al (2017) realizado no Norte do Tocantins, mostra a mesma situação, mesmo as mulheres se esforçando como pescadoras, elas não possuem seu trabalho valorizado, por isso, muitas vezes, elas mesmas não conseguem enxergar a importância do seu papel. Trabalhos com mulheres pescadoras nos demais estados são escassos na literatura.

Os trabalhos realizados com mulheres pescadoras no estado do Pará são recentes e a maioria são trabalhos na versão de artigos científicos, encontrados na plataforma do Google Acadêmico. As pesquisas foram realizadas principalmente em munícipios do Nordeste do paraense, como Bragança, Capanema, Santarém, Alenquer, Barcarena. Tucuruí e na ilha do Marajó e em RESEX marinhas (RESEX São João da ponta; RESEX Caeté/Bragança e RESEX Mãe Curuçá). As plataformas selecionadas apresentam muitos trabalhos acerca do tema, mas a maioria são debates e discussões. Poucos trabalhos mostram a realidade das condições socioeconômicas das mulheres pescadoras no estado.

É possível afirmar que a mulher sempre exerceu papel importante pesca artesanal, tanto na cadeia produtiva pós captura, quanto no beneficiamento do pescado para a comercialização. O trabalho da mulher pescadora ainda é marcado pela invisibilidade e pela falta de reconhecimento, tanto que o termo de "pescadora artesanal" ainda não é muito aceito, pois ainda é uma profissão vista de domínio masculino. A pesquisa mostrou que mesmo as mulheres exercendo várias atividades à pesca, elas não são reconhecidas pela profissão, mas normalmente são representadas pelos maridos e sua atividade é vista apenas como ajuda.

\section{Referências}

Almeida, I.C. (2013). O papel da pesca na eficiência reprodutiva dos ribeirinhos do Baixo Tocantins: $O$ caso do município de Mocajuba - PA. Dissertação (Mestrado em Desenvolvimento Sustentável do Trópico Úmido) - Universidade Federal do Pará, Núcleo de Altos Estudos Amazônicos, Belém.

Araujo, S.P. \& Milani, D. (2017). Reflexões sobre o papel social da mulher catadora de caranguejo da comunidade amazônica de Caratateua, Bragança-PA. In: Anais do Congresso Internacional da Faculdades EST 3: 066-079.

Araujo, Z.T.D.S. (2015). Processo participativo da mulher na cadeia produtiva do mexilhão Mytella charruana (D’orbgny, 1846) na comunidade Vila Nova, Bragança-PA) - Universidade Federal do Pará.

Azevedo, A.K.A. \& Moraes, S.C. (2016). Pesca Artesanal Feminina na Comunidade Porto Grande-São João da Ponta (PA). Universidade e Meio Ambiente, 1(1): 1-13. Disponível em: http://www.reumam.net/index.php/revista/article/view/6/15.

Costa, F.P., Silva, F.N.L., Guedes, A.C.B., Passos, P.H.S., Quadros, M.L.A., Mendonça, R.C., \& Oliveira, L.C. (2020). Mulheres na aquicultura: um estudo de caso no arquipélago do Marajó, Brasil. Research, Society and Development, 9(7), e100973872-e100973872. 
Costa, M.P. \& Futado, G.L. (2015). “Um pesqueiro real”: aspectos organizativos, acesso e uso da pesca na vila do pesqueiro, Soure, Marajó, Pará. In: Anais da Reunião de Antropologia do Norte.

Ferreira M.M., \& Parente, T.G. (2016). Gênero e trabalho das mulheres pescadoras dos reassentamentos rurais no extremo norte do Tocantins. Revista Desafios, 3(esp.), 30-33.

Guedes, A.C.B., Silva, F.N.L., Oliveira, L.A.A., Silva, F.R. \& Sá Barbosa, R. (2021). O papel das mulheres na pesca artesanal no rio Oléria, arquipélago do Marajó, Brasil. Research, Society and Development, 10(1), e31410111816

Lima, B.F. \& Mota, D.M. (2016). Percepção de mulheres extrativistas sobre o trabalho na ilha de MarajóPará. Ideias, 7, 89-110.

Machado, D. (2007). Catadoras de caranguejo e saberes tradicionais na conservação de manguezais da Amazônia brasileira. Revista Estudos Feministas, 15, 485-490.

Oliveira, A.I. (2019). Identidade e território das comunidades tradicionais: como a mulher as pescadora se vê no processo laboral na extração da massa do caranguejo?. Revista Emblemas. 16(2).

Oliveira, B.S.C.; Lima, S.A.A. \& Pinheiro, C.M. (2018). Mercúrio e glutationa em pescadoras do nordeste paraense. Revinter. 11(2): 29-39.

Palheta, M.K.S.; Canete, V.R. \& Cardoso, D.M. (2016). Mulher e mercado: participação e conhecimentos femininos na inserção de novas espécies de pescado no mercado e na dieta alimentar dos pescadores da RESEX Mãe Grande em Curuçá (PA). Boletim do Museu Paraense Emílio Goeldi, 11(3): 601-619.

Pinto, W. (2016). O trabalho invisível das mulheres pescadoras. Jornal da Universidade Federal do Pará. 30(130).

Rocha, A.S.N. (2011). A pesca feminina na comunidade segredinho: Município de Capanema/PA. Dissertação. Universidade Federal do Pará.

Rodrigues, P.C. (2018). Mulheres pescadoras: o significado do trabalho de pesca do camarão na comunidade da salvação, Alenquer/PA. Tese. Universidade Federal do Amazonas.

Sá-Oliveira, J.C., Vasconcelos, H.C.G., Pereira, S.W.M., Isaac-Nahum, V.J. \& Teles-Junior, A.P. (2013). Caracterização da pesca no Reservatório e áreas adjacentes da UHE Coaracy Nunes, Ferreira Gomes, Amapá-Brasil. Biota Amazônia, 3(3): 83-96.

Silva, M.B., Silva, K.C.A., Herrmann, M., Araújo, M.V.L.F. \& Cintra, I.H.A. (2014). Mulheres pescadoras de camarão-da-Amazônia a jusante da usina hidrelétrica de Tucuruí, Amazônia, Brasil. Revista Brasileira de Engenharia de Pesca, 7 (2): 15-33.

Simonian, L.T.L. (2006). Pescadoras de camarão: gênero, mobilização e sustentabilidade na ilha Trambioca, Barcarena, Pará. Boletim do Museu Paraense Emílio Goeldi, 1(2), 35-52.

Sorj, B. (2010). Os cuidados com a família e as desigualdades de gênero e de classe. In: Costa A., Ávila M.B., Soares V., Ferreira V. (Org). Divisão sexual do trabalho, estado e crise do capitalismo. Recife: SOS Corpo, p. 57 - 65.

Sousa, S.L.W. (2018). Mulheres que pescam ou cultivam à margem do lago Maicá, Santarém, Pará: aspectos socioeconômicos, ambientais e de qualidade de vida. Dissertação. Universidade Federal Oeste do Pará.

Sousa, W.L., Monte, O.F.L., Silva, E.R. \& Vieira, A.T. (2018). Protagonismo socioeconômico das pescadoras artesanais do bairro Pérola do Maicá, em Santarém Pará. Revista Ciências da Sociedade, 2(4,): 143-161.

Souza, S.R., Martinez, S.A. \& Gantos, M.C. Mulheres pescadoras: uma análise das produções Bibliográficas acerca das relações de gênero no universo da pesca artesanal. In: Seminário Internacional Fazendo Gênero \& $13^{\circ}$ Women's World Congress. 